\title{
Nonlinear FE analysis of cracks in tension and shear
}

\author{
G. Kesse* and J. M. Lees $\dagger$ \\ Tony Gee and Partners; University of Cambridge
}

\begin{abstract}
Smeared-crack finite element (FE) analyses of reinforced-concrete beams strengthened with prestressed carbonfibre-reinforced polymer (CFRP) shear straps have shown that, at high load levels, the stresses in the straps predicted by the FE analyses were less than the observed experimental values. As the straps are elastic and brittle it is important to investigate this anomaly since otherwise the FE predictions of CFRP shear-strengthened structures could prove to be unconservative. It was postulated that the concrete crack models used in the analyses were inadequate and thus the current work focuses on the FE modelling of cracks subjected to tension and shearing actions. Details of nonlinear FE analyses of the crack behaviour are presented and it is concluded that it is necessary to incorporate advanced crack models in order accurately to predict the stress in the CFRP reinforcing elements.
\end{abstract}

\section{Introduction}

Smeared cracked approaches are commonly used in the nonlinear finite element (NLFE) analysis of concrete. In a smeared-crack formulation, cracked concrete is considered as a continuum and cracked material properties are associated with the element. Advantages of this approach (over a discrete crack model) are faster computation and improved numerical stability. For a brittle nonlinear material such as concrete, however, these finite element models are fundamentally loadhistory dependent and the predicted behaviour relies on the concrete models used to represent the cracked material behaviour. The situation is further complicated by the need to consider the direction of crack opening and the fact that a given crack can be in a state of loading, unloading or reloading.

For ease of incorporation into finite element (FE) code and for general numerical robustness, fairly simple concrete crack models are typically used as the default choice in most commercial FE programs. In many steel-reinforced concrete applications, where the yielding of the steel reinforcement will lead to redis-

\footnotetext{
* Tony Gee and Partners, Esher, Surrey, UK

$\uparrow$ Department of Engineering, University of Cambridge, Trumpington Street, Cambridge, UK

(MCR 61552) Paper received 17 May 2006; accepted 8 December 2006
}

tribution, these models are acceptable and further refinement is not required. This is, however, not necessarily the case in other applications. For example, there has been a growing use of carbon-fibre-reinforced polymers (CFRPs) for the strengthening and repair of existing reinforced-concrete structures. To date, any additional external CFRP reinforcement applied to a structure has typically been passive, but prestressed CFRP systems are also being developed. ${ }^{1}$ Such applications raise a number of considerations including: CFRP is a brittle elastic material; an existing structure may be cracked prior to strengthening; and the addition of any prestress will influence the stress transfer across a crack.

As the CFRP reinforcement does not yield, a knowledge of the true stresses in these reinforcing elements is essential. If the stresses in the CFRP are not correctly modelled, it is possible that the stress predictions will be unconservative. Indeed, smeared-crack FE analyses of reinforced-concrete beams strengthened with external prestressed CFRP vertical shear straps using a constant-beta shear-cracking model (which will be described in greater detail later), underestimated the peak stresses in the CFRP straps and did not predict a strap failure, even when strap failures had been observed experimentally. This major limitation of the analyses was attributed to the crack models used in the FE analyses. ${ }^{2}$ An additional factor is that an existing structure will have been subjected to a complex load history with numerous loading, unloading and reload- 
ing phases. If the concrete has cracked, then residual normal and/or shear displacements would be expected. In most commercial FE codes, the default unloading and reloading crack models do not include any residual deformations. Furthermore, if the external CFRP reinforcement is prestressed, it would be expected that the prestress force would help to close any existing shear cracks and thereby reduce the crack widths. As the beam is subjected to further loading/unloading and reloading cycles, the reinforcement would also affect the stress transfer between the CFRP reinforcement and the cracked concrete as cracks open and close.

To investigate the influence of different crack models on the predicted behaviour, normal and shear cracking models that incorporate the nonlinear behaviour of cracks and also loading, unloading and reloading are considered in the current paper. The aim of undertaking these comparisons was to help to explain the potential cause of the underestimation of the maximum strap stress predictions in the FE analyses of reinforcedconcrete beams strengthened with prestressed CFRP straps $^{2}$ and provide guidance for the selection of appropriate concrete crack models.

\section{Crack models}

Normal crack models, where the crack opening is perpendicular to the crack, and shear crack models, where the relative movement is along the crack axis, have been developed. Typically, experiments to observe the normal or shear crack behaviour are carried out and general numerical material models are then formulated using parameters such as the concrete strength properties or aggregate size.

In the most comprehensive case, a crack model would cover the entire loading regime. This would include the formation of new cracks (loading), crack closing (unloading) and the reopening of existing cracks (reloading). As will be discussed, however, some models have only been developed for a particular subset of loading stages.

\section{Normal crack models}

Experimental studies. Evans and Marathe are credited by Bazant and Planas ${ }^{3}$ with developing probably the oldest experiments designed to measure the stress strain behaviour of concrete in direct tension. More recently, a number of further studies ${ }^{4-7}$ have all furnished information on the softening behaviour of cracks.

Important parameters that have evolved from the experimental studies are the shape of the softening curve and the fracture energy. The softening curve represents the relationship between the applied force and displacement quantities (which can also be expressed as stress versus displacement, stress versus strain, etc.) during loading. The fracture energy, $G_{f}$, represents the energy consumed in forming the crack.

In order to consider the unloading and reloading behaviour, cyclic tests are required. The number of cyclic experimental studies, where a specimen is subjected to a number of unloading and reloading cycles at various loading stages, is however somewhat more limited than studies where a specimen is loaded monotonically to failure. Work by Gopalaratnam and Shah ${ }^{6}$ and Yankelevsky and Reinhardt ${ }^{8}$ has provided information on the cyclic behaviour of concrete and typical results ${ }^{8}$ show that the monotonic curve forms an envelope from which unloading starts (see Fig. 1). The unloading curve is highly nonlinear, there are residual deformations when the specimen is unloaded and the amount of residual deformation at zero stress depends on the level of maximum strain attained.

Numerical formulation. Different shapes of loading (softening) curves for cracks in normal tension have been proposed, namely linear or nonlinear softening models. ${ }^{5}$ For ease of incorporation into FE models, $G_{\mathrm{f}}$ is typically related to the concrete compressive or tensile strength. From the assumed shape of the softening curve and a knowledge of $G_{\mathrm{f}}$, the loading curve can then be developed.

To model the cyclic response, Yankelevsky and Reinhardt formulated a normal crack model that tracks the unloading and reloading curve. ${ }^{4}$ The shape of the model is shown schematically in Fig. 2. The model incorporates a rather complex nonlinear response during unloading and reloading which leads to permanent deformations and is consistent with what is observed experimentally (see Fig. 1).

The procedure for generating the unloading or reloading curve starts by establishing defined critical stress points, known as focal points, based on the tensile strength of the concrete. The corresponding displacement values for the focal points are obtained

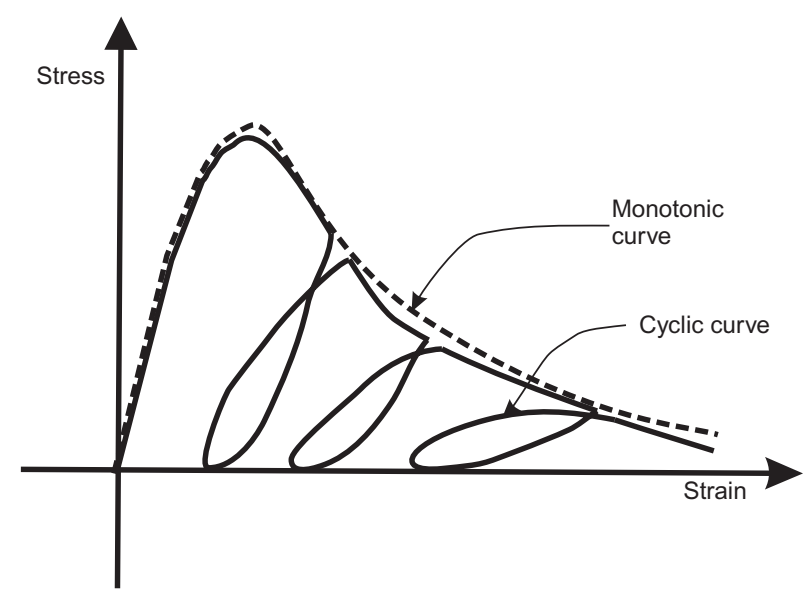

Fig. 1. Schematic example of monotonic and cyclic response of normal cracks after Ref [8]

Magazine of Concrete Research, 2007, 59, No. 4 


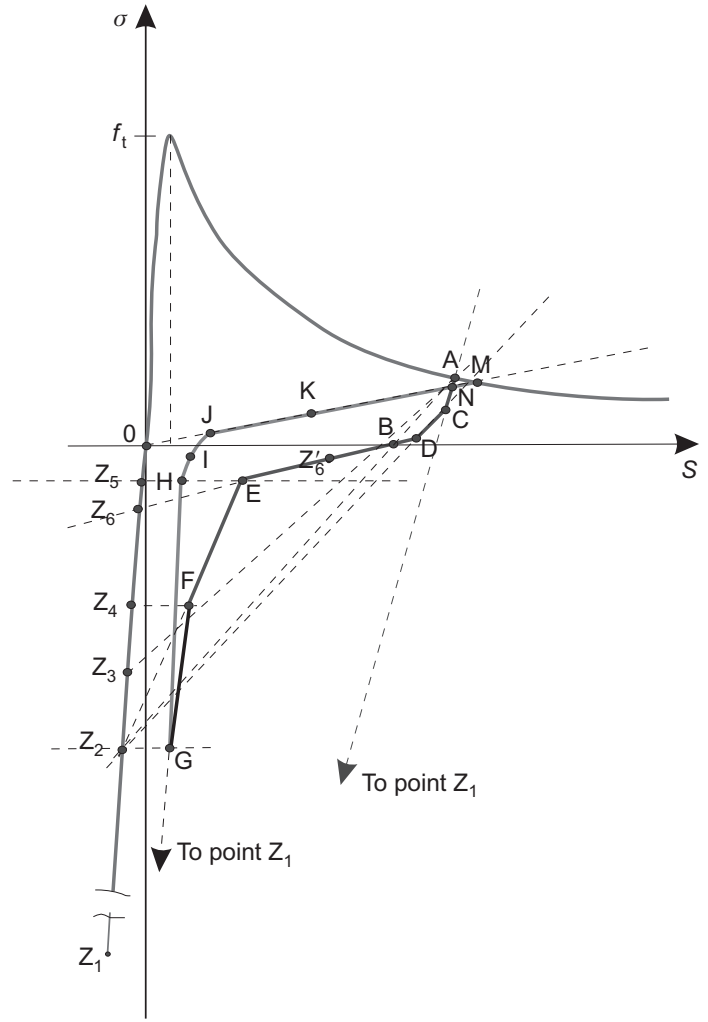

Fig. 2. Normal crack unloading and reloading model

using the stress and the Young's modulus of elasticity of concrete in compression. The mapping of the unloading and reloading curves then requires various geometric constructions using the focal points and intersections with the axes (see Ref. [4] for further details).

\section{Shear crack models}

Experimental studies. The testing of cracked concrete in shear has also been carried out. ${ }^{9-15}$ In these tests, normal cracks are initially formed and then sheared (a typical test set-up is shown in Fig. 3). Depending on the experimental set-up and procedure, the main parameters considered are the crack width, concrete strength, aggregate size, surface roughness of the crack plane and restraint provided by any reinforcement crossing the crack.

Typical results show that the shear stiffness is mainly a function of the normal crack opening ${ }^{9,10}$ and that the aggregate size and shape of aggregate seem to have very little influence on the behaviour. ${ }^{9}$ The level of restraint, which to an extent is defined by the test procedure, is also important and, for example, compressive stresses develop if the crack is not allowed to dilate. Tests where the perpendicular stress is zero ${ }^{11}$ or the ratio of crack opening to shear displacement is kept constant $^{15}$ have been conducted. A further variation is to fix the normal stress perpendicular to the shear

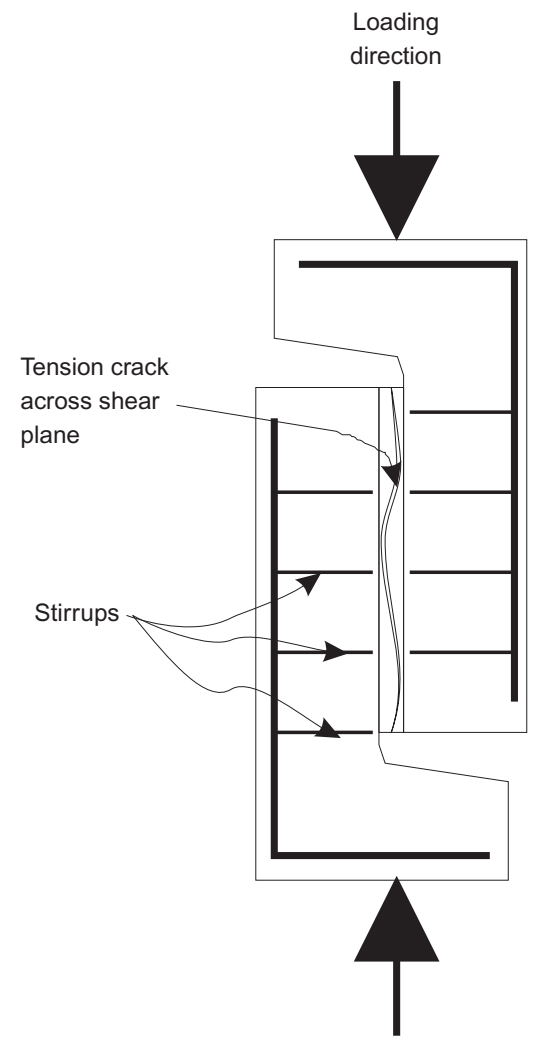

Fig. 3. Shear test

cracks and then allow the normal crack width to increase during shearing. ${ }^{16}$

Cyclic shear tests have also been performed, for example Refs $[9,16,17]$, and the experimental set-up is similar to that used for monotonic testing. Failure under cyclic loading is no different from that experienced in monotonic loading and depends on the crack width. ${ }^{9}$ The major difference is the accumulation of residual shear displacements with progressive loading. The crack surfaces sustain more damage with repeated loading. In particular, any surface irregularities are worn down while the aggregate tends to become rounded.

Numerical formulation. Unfortunately it was not possible to find a single nonlinear shear crack model that was applicable for all stages of loading/unloading and reloading. Thus, in the current work, a nonlinear loading model ${ }^{12}$ was combined with a nonlinear unloading/reloading model. ${ }^{17}$

Yoshikawa et al. ${ }^{12}$ formulated a comprehensive model for shear crack loading using the following relationship between the shear stress, $\tau_{\mathrm{nt}}^{c}$, the normal stress, $\sigma_{\mathrm{n}}^{c}$, the shear displacement, $\delta_{\mathrm{t}}$, and the normal crack opening, $\delta_{\mathrm{n}}$

$$
\left\{\begin{array}{l}
\mathrm{d} \tau_{\mathrm{nt}}^{c} \\
\mathrm{~d} \sigma_{\mathrm{n}}^{c}
\end{array}\right\}=k_{\mathrm{t}}\left[\begin{array}{cc}
1 & -(1-\xi) / \beta_{\mathrm{d}} \\
-1 / \mu_{\mathrm{f}} & 1 /\left(\mu_{\mathrm{f}} \beta_{\mathrm{d}}\right)
\end{array}\right]\left\{\begin{array}{l}
\mathrm{d} \delta_{\mathrm{t}} \\
\mathrm{d} \delta_{\mathrm{n}}
\end{array}\right\}
$$

where 


$$
\begin{gathered}
k_{\mathrm{t}}=\frac{\partial \tau_{\mathrm{nt}}^{c}}{\partial \delta_{\mathrm{t}}} \quad k_{\mathrm{n}}=\frac{\partial \sigma_{\mathrm{n}}^{c}}{\partial \delta_{\mathrm{n}}} \quad \mu_{\mathrm{f}}=-\frac{\partial \tau_{\mathrm{nt}}^{c}}{\partial \sigma_{\mathrm{n}}^{c}} \\
\beta_{\mathrm{d}}=\left(\frac{\partial \delta_{\mathrm{n}}}{\partial \delta_{\mathrm{t}}}\right) \quad \xi=\mu_{\mathrm{f}} \beta_{\mathrm{d}} k_{\mathrm{n}} / k_{\mathrm{t}}
\end{gathered}
$$

and $k_{\mathrm{t}}=$ the shear stiffness; $k_{\mathrm{n}}=$ the normal stiffness; $\mu_{\mathrm{f}}=$ the frictional coefficient and $\beta_{\mathrm{d}}=$ the dilatancy ratio.

A further loading model was proposed by Heuze and Barbour $^{18}$ who used a more physical approach by considering a shear crack that is restrained (as shown in Fig. 4). When such a crack is sheared $\left(\delta_{t}\right)$, the two blocks will override causing an increase in the crack opening $\left(\delta_{\mathrm{n}}\right)$. At the same time the blocks will be pushed back by the boundary restraints and thus these compressive forces will attempt to close the crack. The normal opening $\delta_{\mathrm{n}}$ is taken to be a function of the shear displacement $\delta_{\mathrm{t}}$ and the normal stress $\sigma_{\mathrm{n}}^{c}$, but the shear stress $\tau_{\mathrm{nt}}^{c}$ is a function of the shear displacement $\delta_{t}$ only. This decoupling of the dilatancy effects leads to a much simpler relationship where the final matrix is a special case of Yoshikawa et al.'s framework with $\xi=1$.

$$
\left\{\begin{array}{l}
\mathrm{d} \sigma_{\mathrm{n}}^{c} \\
\mathrm{~d} \tau_{\mathrm{nt}}^{c}
\end{array}\right\}=k_{\mathrm{t}}\left[\begin{array}{cc}
1 /\left(\mu_{\mathrm{f}} \beta_{\mathrm{d}}\right) & -1 / \mu_{\mathrm{f}} \\
0 & 1
\end{array}\right]\left\{\begin{array}{l}
\partial \delta_{\mathrm{n}} \\
\partial \delta_{\mathrm{t}}
\end{array}\right\}
$$

Note that the matrix has been reordered to be consistent with the convention used in the derivations to follow.

For shear crack unloading and reloading, the model developed by Jimenez et al. ${ }^{17}$ was incorporated. The model can be described using Fig. 5 where the idealised load-shear displacement is shown.

The unloading follows a bilinear curve until reloading starts or loading in the opposite sense is activated. The residual displacement exhibited when the specimen in unloaded is expressed as a fraction $\omega$ of the maximum shear displacement. The shear stress and displacement at which the change in reloading stiffness occurs is expressed as a proportion $\theta_{\mathrm{s}}$ of the shear stress $\tau$ and a proportion $\Omega$ of the shear displacement $\delta_{\mathrm{t}}$ associated with $\tau$. Upon unloading, a residual slip will result.

For a typical shear crack, the resulting combined

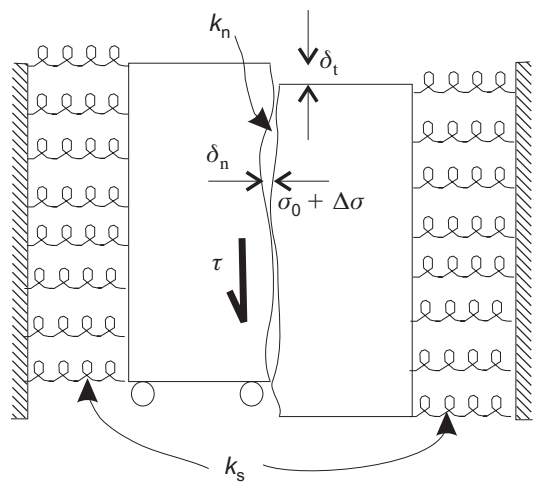

Fig. 4. Restrained shear crack

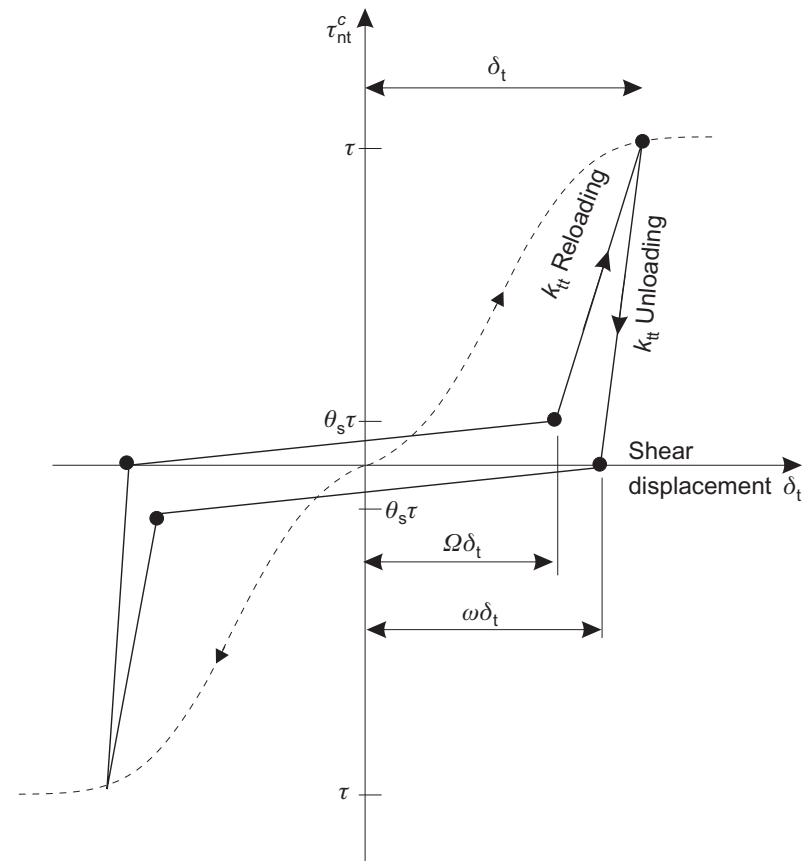

Fig. 5. Shear unloading and reloading model

loading model ${ }^{12}$ and unloading/reloading $\operatorname{model}^{17}$ is shown schematically in Fig. 6.

\section{Important features}

For both normal and shear crack loading, it appears that the monotonic loading envelope is broadly similar to the cyclic loading envelope. The key difference is that when a specimen is unloaded and reloaded in a cyclic fashion, residual displacements are manifested during unloading and the stiffness on reloading reduces as damage accumulates.

It is thus the treatment of unloading and reloading and residual deformations that is of most interest and will be the focus of the current work.

\section{Smeared-crack FE formulation}

Normal and shear crack behaviour are two primary sources of nonlinear effects in RC structures and need

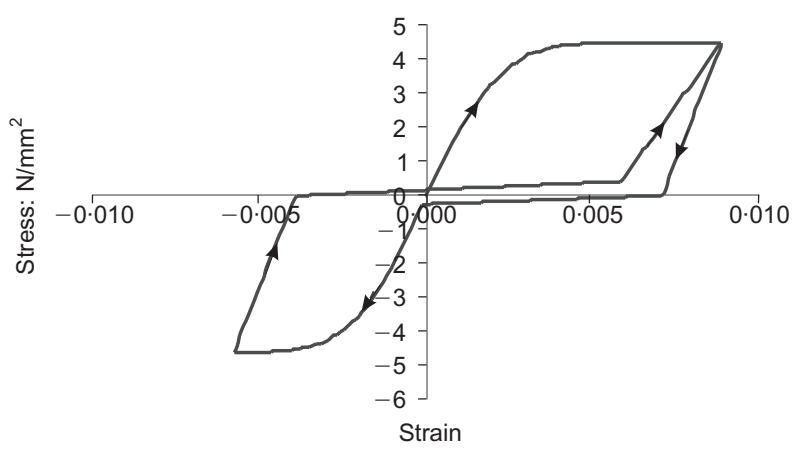

Fig. 6. Shear loading, unloading and reloading model

Magazine of Concrete Research, 2007, 59, No. 4 
to be incorporated in FE programs if meaningful results are to be obtained. In the following, the crack stiffness derivation proposed by de Borst ${ }^{19}$ will be summarised. The representation of cracks in a smeared-crack analysis will also be highlighted.

Consider an element with a crack band $c_{\mathrm{b}}$ shown in Fig. 7. The global axes are denoted, $(x, y)$ and the local axes, $(t, n)$ are aligned with the crack of angle $\theta$ measured anticlockwise with respect to the global $x$ axis. The change in total strain $\boldsymbol{d} \boldsymbol{\varepsilon}$ within the crack band in the global direction can be decomposed into the change in elastic strain $\boldsymbol{d} \boldsymbol{\varepsilon}^{\boldsymbol{e}}$ and the change in fracture strain $d \varepsilon^{f}$

$$
d \varepsilon=d \varepsilon^{e}+d \varepsilon^{f}
$$

In matrix form, equation (4) becomes (two-dimensional plane stress analysis)

$$
\left\{\begin{array}{c}
\mathrm{d} \varepsilon_{x} \\
\mathrm{~d} \varepsilon_{y} \\
\mathrm{~d} \varepsilon_{x y}
\end{array}\right\}=\left\{\begin{array}{c}
\mathrm{d} \varepsilon_{x}^{e} \\
\mathrm{~d} \varepsilon_{y}^{e} \\
\mathrm{~d} \varepsilon_{x y}^{e}
\end{array}\right\}+\left\{\begin{array}{c}
\mathrm{d} \varepsilon_{x}^{f} \\
\mathrm{~d} \varepsilon_{y}^{f} \\
\mathrm{~d} \varepsilon_{x y}^{f}
\end{array}\right\}
$$

The change in local fracture strains $\boldsymbol{d e} \boldsymbol{e}^{\boldsymbol{f}}$ (i.e. d $e_{\mathrm{t}}$ and $\left.\mathrm{d} e_{\mathrm{n}}\right)$ of a particular crack of angle $\theta$ can be written with respect to the change in global crack strain $\boldsymbol{d} \varepsilon^{f}$ as

$$
\boldsymbol{d} \boldsymbol{\varepsilon}^{f}=\boldsymbol{N} \boldsymbol{d} \boldsymbol{e}^{f} \text { where } \boldsymbol{d} \boldsymbol{e}^{f}=\left\{\begin{array}{l}
\mathrm{d} e_{\mathrm{n}} \\
\mathrm{d} e_{\mathrm{t}}
\end{array}\right\}
$$

where $\boldsymbol{N}$ is the crack angle transformation matrix. For multiple cracks at the same integration point, equation (6) becomes

$$
d \varepsilon^{f}=N_{1} d e_{1}^{f}+N_{2} d e_{2}^{f}+N_{3} d e_{3}^{f}+\ldots+N_{i} d e_{i}^{f}
$$

where each $N_{i}$ is the crack angle matrix of each particular crack. The local crack stress $\boldsymbol{d} \boldsymbol{s}$ on the crack is related to the global change in stress as

$$
d s=N^{T} d \sigma
$$

$$
\text { where } \boldsymbol{d} \boldsymbol{s}=\left\{\begin{array}{l}
\mathrm{d} s_{\mathrm{n}} \\
\mathrm{d} s_{\mathrm{t}}
\end{array}\right\} \text { and } \boldsymbol{d} \boldsymbol{\sigma}=\left\{\begin{array}{l}
\mathrm{d} \sigma_{x} \\
\mathrm{~d} \sigma_{y} \\
\mathrm{~d} \sigma_{x y}
\end{array}\right\}
$$
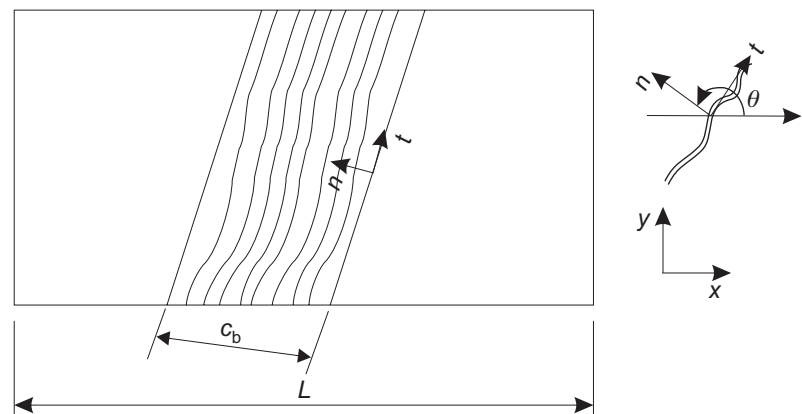

Fig. 7. Crack band width
The constitutive relationship between the local crack stresses and local crack strains is given by

$$
d s=D^{c r} d e^{f}
$$

where $D^{c r}$ is the cracked stiffness matrix. The change in stress in the global direction is also related to the global elastic strains by

$$
d \sigma=D^{e} d \varepsilon^{e}
$$

where $\boldsymbol{D}^{\boldsymbol{e}}$ is the elastic stiffness matrix. Substituting equations (9) and (10) into equation (8) and using equations (4) and (6) leads to

$$
d e^{f}=\left[D^{c r}+N^{T} D^{e} N\right]^{-1} N^{T} D^{e} d \varepsilon
$$

Combining equations (4), (6), (10) and (11) results in an expression for $\boldsymbol{d} \boldsymbol{\sigma}$

$$
d \sigma=\left[D^{e}-D^{e} N\left(D^{c r}+N^{T} D^{e} N\right)^{-1} N^{T} D^{e}\right] d \varepsilon
$$

The change in global strain is therefore related to the change in the global stress resulting in the constitutive relation for cracked concrete.

The $\boldsymbol{D}^{\boldsymbol{c} r}$ matrix introduced in equation (9) can be thought of as being made up of the normal crack stiffness $\boldsymbol{D}_{n}^{c r}$ and the shear crack stiffness, $\boldsymbol{D}_{t}^{c r}$ i.e. $D^{c r}=D_{n}^{c r}+D_{t}^{c r}$. For normal crack opening, the $D_{n}^{c r}$ matrix has the form

$$
\boldsymbol{D}_{n}^{c r}=\left[\begin{array}{cc}
k_{\mathrm{c}} & 0 \\
0 & 0
\end{array}\right]
$$

where $k_{\mathrm{c}}$ is the softening modulus. For a shear crack, the $D_{t}^{c r}$ matrix has the form

$$
\boldsymbol{D}_{\mathrm{t}}^{\boldsymbol{c r}}=\left[\begin{array}{ll}
k_{\mathrm{nn}} & k_{\mathrm{nt}} \\
k_{\mathrm{tn}} & k_{\mathrm{tt}}
\end{array}\right]
$$

It is the constituent terms of the $D_{n}^{c r}$ and $D_{t}^{c r}$ crack matrices, that is, $k_{\mathrm{c}}, k_{\mathrm{nn}}, k_{\mathrm{nt}}, k_{\mathrm{tn}}$ and $k_{\mathrm{tt}}$ which are required in order to carry out an FE analysis. The experiments and numerical models described in the section on crack models have shown that these parameters are not constant, but depend on the width of a crack and also the stage of loading. The modelling of the crack behaviour is thus complex since a given crack can be in state of loading, unloading or reloading either in tension or in shear and appropriate parameters need to be used to reflect this behaviour. Moreover, in commercial FE packages such as DIANA, ${ }^{20}$ the FE program used in the current work, the ability to incorporate advanced crack models is limited. It was therefore necessary to write a finite element programme (CamFEA), a visual $\mathrm{C}++$ object-oriented programme, to consider a wider range of crack models. ${ }^{2}$

In the following, the FE predictions using advanced crack models in CamFEA are compared with those using the simpler default crack models found in a commercial FE software program. As the emphasis is to compare the behaviour of different models and how 
they influence the stress transfer, the FE meshes used in the examples are relatively simple.

\section{Normal crack matrix: $D_{n}^{c r}$}

The modulus coefficient $k_{\mathrm{c}}$ in equation (13) refers to the tangent of the curve of stress versus crack fracture strain. However, in most experiments it is the total strain $\boldsymbol{d} \boldsymbol{\varepsilon}$ that is measured and the elastic strains must be subtracted before the modulus can be determined and used. If concrete is considered as a brittle material with no softening behaviour then $k_{\mathrm{c}}=0$. However, for the modelling of tension softening $k_{\mathrm{c}}$ must be assigned an appropriate value.

\section{Normal crack models}

In the current work, only a linear loading (softening) model will be considered since, as discussed, the feature of most interest in the normal crack representation was not the shape of the loading curve but the treatment of unloading and reloading and the residual deformations. The linear softening model was combined with two different unloading/reloading models (denoted models (a) and (b)).

Normal crack model (a): linear softening with nonlinear unloading and reloading and residual deformations. Yankelevsky and Reinhardt's ${ }^{4}$ numerical model mimics the experimentally observed behaviour when normal cracks are unloaded and reloaded. The relationship between $\mathrm{d} s_{\mathrm{n}}$ and $\mathrm{d} e_{\mathrm{n}}$ (which represents the coefficient $k_{\mathrm{c}}$ ) can be inferred from curves such as those shown schematically in Fig. 2. This relationship was therefore programmed into CamFEA. The model includes nonlinear unloading/reloading and takes into account residual deformations.

Normal crack model (b): linear softening with linear unloading and reloading and no residual deformations. A linear unloading/reloading model with no residual deformations was the default choice in the commercial FE program used. The advantage was that linear softening without permanent deformations is fairly straightforward and robust. Once a crack forms, the softening gradient is used. If the crack is loading or unloading, a secant gradient from the location of unloading is followed. This model does not, however, include any residual deformations and thus does not fully represent the experimental trends (see Fig. 1).

\section{FE model: benchmarking example}

A simple example was used to compare the behaviour of the concrete models. The properties of the concrete are given in Table 1 . The fracture energy, $G_{\mathrm{f}}=$ $0.125 \mathrm{~N} / \mathrm{mm}$ used in the linear softening model was estimated such that a crack opening of $0 \cdot 1 \mathrm{~mm}$ will result in no stress transfer.

Figure 8(a) shows a single unreinforced-concrete finite element. The element is loaded and unloaded in tension by forces $F_{\mathrm{h}}$. Fig. 8(b) shows the same element but with the addition of an internal steel reinforcing bar with perfect bond.

\section{FE results}

In Fig. 9 it can be seen that the unloading and reloading stiffness results for the unreinforced element reflect the input models. The results obtained using crack model (b) (with no permanent deformations) differ from those of crack model (a). The implications are that if permanent deformations are not modelled, not only will the residual deformations be incorrect but also the reloading stiffness of the structure will not be correctly predicted.

If the element is reinforced with an internal reinforcing bar, then the existence of permanent deformations will affect the residual stress in the reinforcement. Fig. 10(a) shows the load-displacement response for the reinforced model after loading and unloading to its initial state. The stresses in the reinforcement are shown in Fig. 10(b). With model (b), the residual stress in the reinforcement is zero and the structure unloads to its original state and all the cracks have closed. With model (a), in the state where there is no external load, there are still residual stresses in the reinforcement with some permanent crack openings. Additional forces would be required to completely close the cracks. In effect, although the external load applied might be the same, the state of stress in the concrete and the rein-

Table 1. Input for FE analysis

\begin{tabular}{|c|c|}
\hline \multicolumn{2}{|l|}{ Concrete } \\
\hline $\begin{array}{l}\text { Mesh width } \\
\text { Thickness } \\
\text { Aggregate size } \\
\text { Crack band width } \\
f_{\mathrm{t}} \\
f_{\mathrm{c}} \\
G_{\mathrm{f}} \\
\text { Young's modulus } \\
\beta_{\text {sr }}\end{array}$ & $\begin{array}{l}40 \mathrm{~mm}-4 \times \text { aggregate size } \\
1 \mathrm{~mm} \\
10 \mathrm{~mm} \\
40 \mathrm{~mm}-\text { equal to element width } \\
2 \cdot 5 \mathrm{~N} / \mathrm{mm}^{2} \\
40 \mathrm{~N} / \mathrm{mm}^{2} \\
0 \cdot 125 \mathrm{~N} / \mathrm{mm}^{2} \text { (linear softening models) } \\
20000 \mathrm{~N} / \mathrm{mm}^{2} \\
0 \cdot 2 \text { (for Beta models) }\end{array}$ \\
\hline \multicolumn{2}{|l|}{ Steel reinforcement } \\
\hline $\begin{array}{l}\text { Area } \\
\text { Young's modulus }\end{array}$ & $\begin{array}{l}1 \mathrm{~mm}^{2} \\
200000 \mathrm{~N} / \mathrm{mm}^{2}\end{array}$ \\
\hline \multicolumn{2}{|c|}{ Strap reinforcement } \\
\hline $\begin{array}{l}\text { Area } \\
\text { Young's modulus }\end{array}$ & $\begin{array}{l}2 \mathrm{~mm}^{2} \\
130000 \mathrm{~N} / \mathrm{mm}^{2}\end{array}$ \\
\hline
\end{tabular}

Magazine of Concrete Research, 2007, 59, No. 4 

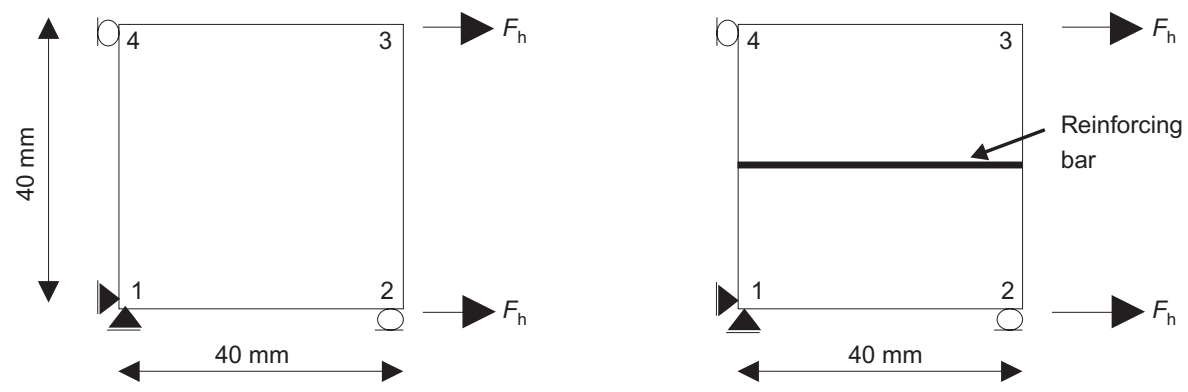

Fig. 8. Single element for uniaxial tension

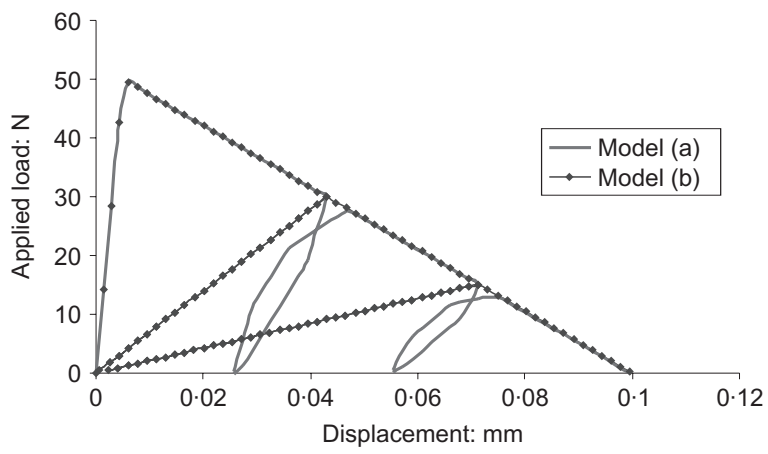

crack model (a) will be used in all the examples henceforth so that the influence of residual normal deformations on the shear crack results can be observed.

\section{Shear crack models: $D_{t}^{c r}$}

\section{Concrete models}

Both nonlinear and linear shear crack models were considered.

Nonlinear shear crack model - (NL model). The

Fig. 9. Comparison of normal crack models

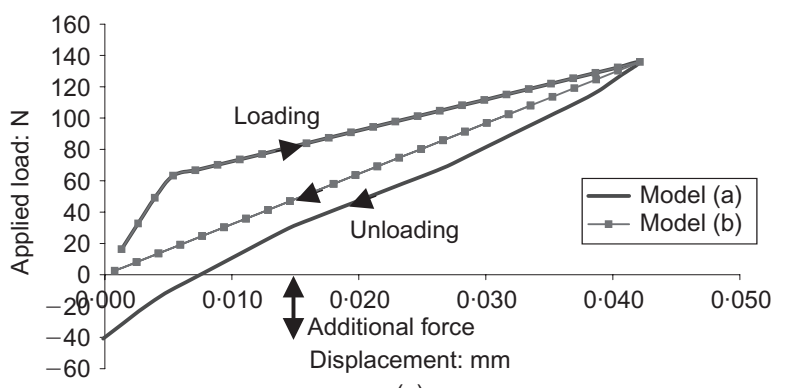

(a)

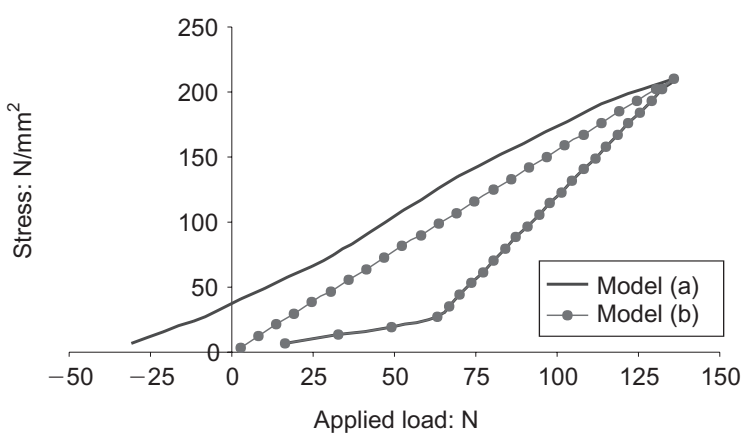

(b)

Fig. 10. Normal crack loading and unloading: (a) loaddisplacement behaviour; (b) comparison of reinforcement stresses

forcement crossing the cracks may be quite different depending on the models used in the analysis.

Since the influence of partially closed cracks on the shear behaviour will be of particular interest, normal cracked shear stiffness matrix, where the shear stress is decoupled from the normal displacement and hence $k_{\mathrm{tn}}=0$ [equation (3)] can be represented as

$$
\boldsymbol{D}_{t}^{c r}=\left[\begin{array}{cc}
k_{\mathrm{nn}} & k_{\mathrm{nt}} \\
0 & k_{\mathrm{tt}}
\end{array}\right]
$$

Again, the matrix coefficients can be deduced from experimental results such as those described in the above section on shear crack models and the combined loading model ${ }^{12}$ and unloading/reloading model ${ }^{17}$ shown schematically in Fig. 6 was implemented into CamFEA.

For the loading curve, the expressions for the various coefficients proposed by Yoshikawa et al. were implemented. Yoshikawa et al. calibrated their model using data from relevant experiments in the literature (please see Ref. [12] for further details).

For the unloading and reloading branch, the average values selected for the coefficients $\theta_{\mathrm{s}}$ and $\Omega$ were $0 \cdot 1$ and 0.7 respectively (see Fig. 5). These values were based on experimental results by Jimenez et al. ${ }^{17}$

Linear shear crack constant-beta model: loading, unloading and reloading. The use of a constant-beta shear crack model is a common option in FE programs. A constant-beta model treats loading, unloading and reloading as the same process by introducing a shear retention factor $\beta_{\mathrm{sr}}$, that is multiplied by the elastic shear modulus to represent the cracked shear modulus. It is thus very simple and straightforward and presents no difficulties in terms of formulating the cracked stiffness matrix. In matrix form, all the coefficients of $\boldsymbol{D}_{t}^{c r}$ are set to zero except $k_{\mathrm{tt}}$, which 
is equated to $\beta_{\mathrm{sr}} G$ where $G$ is the elastic shear modulus, that is

$$
\boldsymbol{D}_{\boldsymbol{t}}^{\boldsymbol{c r}}=\left[\begin{array}{cc}
0 & 0 \\
0 & \beta_{\mathrm{sr}} G
\end{array}\right]
$$

There remains the question of how to select an appropriate value of $\beta_{\mathrm{sr}}$. In the early stages of loading, a fairly high value is likely to be appropriate. As more and more cracking occurs, however, the shear stiffness will drop and thus $\beta_{\text {sr }}$ should decrease. In more advanced beta crack models, the value of beta is thus a function of the crack width. A constant value of $\beta_{\text {sr }}$ of $0 \cdot 2$ is used here but the best choice of beta will depend on the problem and, in the absence of experimental calibration, it is difficult to determine an appropriate value.

\section{FE model: benchmarking example}

The three concrete-element FE mesh shown in Fig. 11 was used to analyse cracks subjected to shear forces. The elements labelled 1 and 3 are both elastic and do not crack whilst element 2 has the properties shown in Table 1 . The analysis commences by displacing the mesh horizontally so that tensile stresses develop and a normal crack forms in element 2 . The crack formed in element 2 is then subjected to shear stresses by displacing element 3 vertically.

To investigate the influence of normal crack opening on the shear behaviour, two different cases were considered. In the first case, the structure was displaced up to a level corresponding to a normal strain of 0.001 (cr1). A shear displacement was then applied. In the second case, the normal crack strain was approximately 0.002 (cr2) prior to the application of the shear displacement. The analysis was therefore performed under variable end restraint.

\section{FE results}

Figure 12 shows the applied shear load versus shear displacement for both the constant-beta model (Beta) and the nonlinear model (NL). The constant-beta model predicted that the stiffness decreased as a result of a larger initial normal crack strain but, irrespective of the normal crack width at which shearing commenced, the peak load predictions were similar. Furthermore, it also did not predict a maximum shear stress that the con-

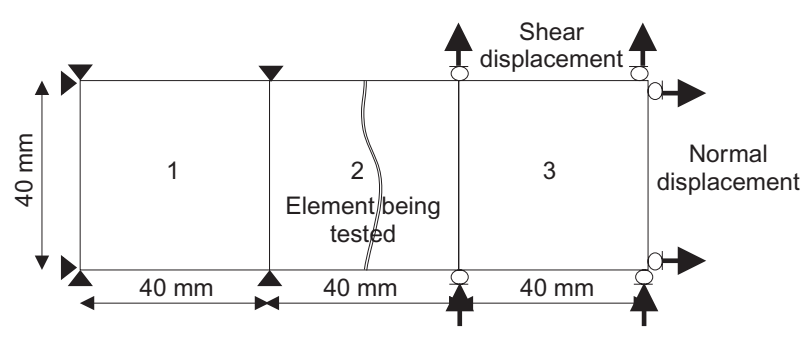

Fig. 11. FE mesh for shear test

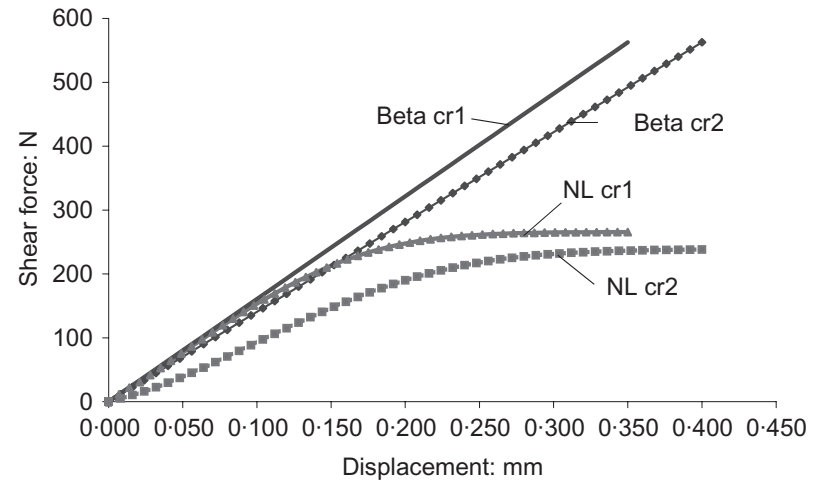

Fig. 12. Comparison of shear behaviour

crete could carry and hence would not be able to predict failure unless some other mechanism is present.

In contrast, the nonlinear model results in Fig. 12 show that both the stiffness and peak load depend on the normal crack opening. The bigger the initial crack opening, the smaller the element stiffness. The stiffness was also not constant but varied with shear slip. The model tended to predict a maximum shear stress that the cracked element could carry and thus could predict failure owing to the loss of aggregate interlock. Note that the results shown were all stopped after a specified number of iterations when the main differences had been observed. The normal stress at a typical integration point of element 2 is shown in Fig. 13. In the constant-beta model there is no change in the normal stress after shearing commenced while the nonlinear model predicts a drop in the tensile stress carried across the crack. Eventually compressive forces develop across this interface.

If the constant-beta model is used, it is assumed that the concrete will always carry a constant portion of the shear force and the remainder of the shear force will be carried by other elements. In the nonlinear model, however, the shear loads depend on the crack opening and ultimately an element will be unable to sustain further loads thus transferring most of the load to the other load-carrying members.

The stiffness variation is also expected to influence the overall structural behaviour. In the nonlinear model, the stiffness degrades as the shearing force and crack widths increase while in the beta model a constant stiffness is maintained. The implications of this might be that the stiffness of the structure would not change to reflect crack widening and increased shearing as the applied loads are increased.

In order for the constant-beta model to predict failure, multiple cracks must be allowed to form at an integration point. The fixed single crack model used in the analysis becomes less accurate as the principal stress directions rotate. Allowing multiple cracks to form will lead to a reduction in the stiffness terms leading to an unstable element (see equation (11)). The difficulties in this approach are therefore in determin- 


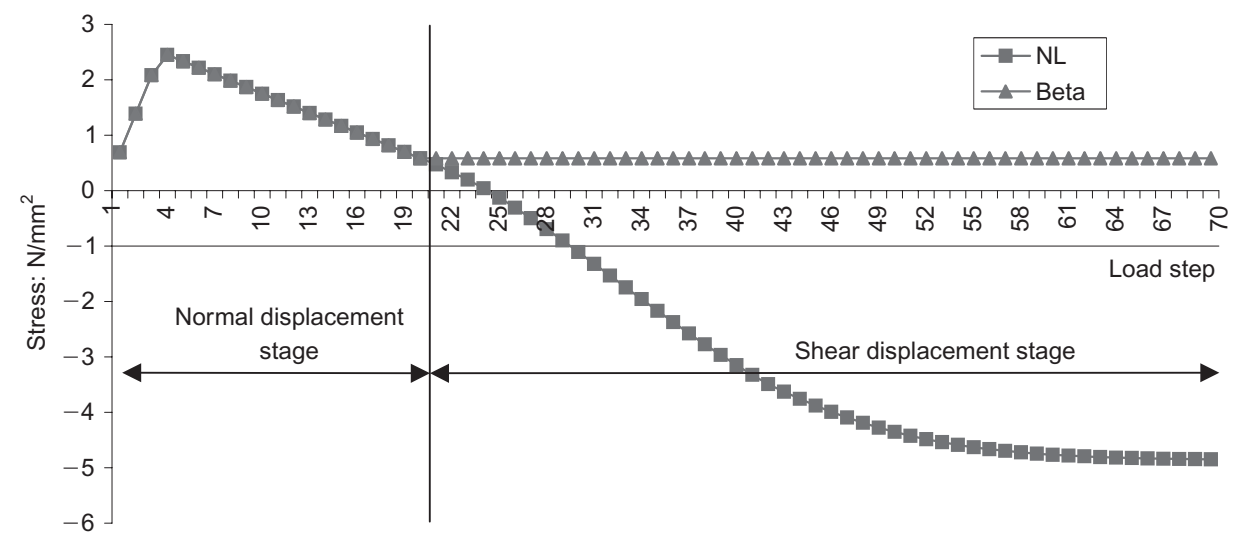

Fig. 13. Comparison of normal stress across a crack

ing the criteria for multiple cracking and also the possibility of creating a spurious failure mechanism if the cracked stiffness matrix becomes indeterminate. ${ }^{21}$

\section{Influence of prestressed CFRP strap on concrete shear behaviour}

Once the concrete models of interest were developed, it was possible to consider the influence of a prestressed elastic CFRP reinforcing element on the crack behaviour. In an existing structure requiring shear strengthening, cracks might exist before the vertical unbonded CFRP straps are incorporated. These cracks will normally be partially unloaded (dead loads would remain) and then the shear strap prestressed before the structure is subjected to additional loading. Even in beams where shear cracks form, normal crack opening occurs first followed by shearing. ${ }^{3}$

A model to mimic this sequence was developed (see Fig. 14) using the input parameters shown in Table 1. The horizontal forces $F_{\mathrm{h}}$, are applied first in order for a crack to form. The direction of these forces is then reversed such that the value of $F_{\mathrm{h}}$ is zero, that is, there is no external load but some residual deformations exist. The unbonded strap is then prestressed and the shear forces $F_{\mathrm{v}}$ applied until failure or the analysis was stopped. Note that if the initial prestress level is varied, the extent of crack closure will also vary. The applied

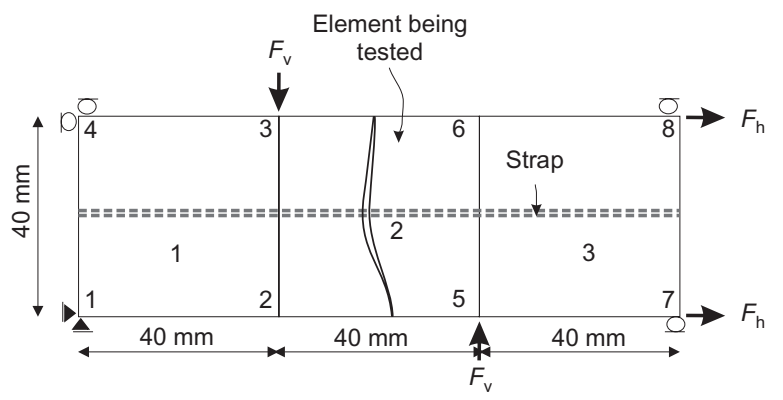

Fig. 14. FE mesh with additional strap shear force $F_{\mathrm{v}}$ and the vertical displacement at node 5 are used as the reference force and displacement respectively for discussing the load-displacement plots.

Figure 15 shows the load-displacement plots for the case where the initial prestress level was varied (125, 130 and $135 \mathrm{MPa}$ ) but the cross-sectional area of the reinforcement was constant $\left(2 \mathrm{~mm}^{2}\right)$. Two different shear models were used for the cracks (the constantbeta model (Beta) and the NL model). The NL model predicts a response with a varying stiffness and also a peak shear force. The stiffness and the peak load both increase with an increasing prestress force. The Beta model on the other hand predicts a linear response with only a minor change in stiffness even as the shearing strain increases drastically. The various levels of prestress did not seem to influence the maximum shear load (the analysis was stopped at the values shown in the plot).

Figure 16 shows the stress in the strap during the shearing stage. The NL model predicted a nonlinear rise in the stress in the strap. The rate of change of stress was inversely related to the prestress level. Initially the stress in the strap NL-135P was the highest but this reversed at a certain stage such that the stress in strap NL-125P became the highest. There is also a drastic increase in the stress gain at stresses above around $225 \mathrm{MPa}$. In combination with Fig. 17, which

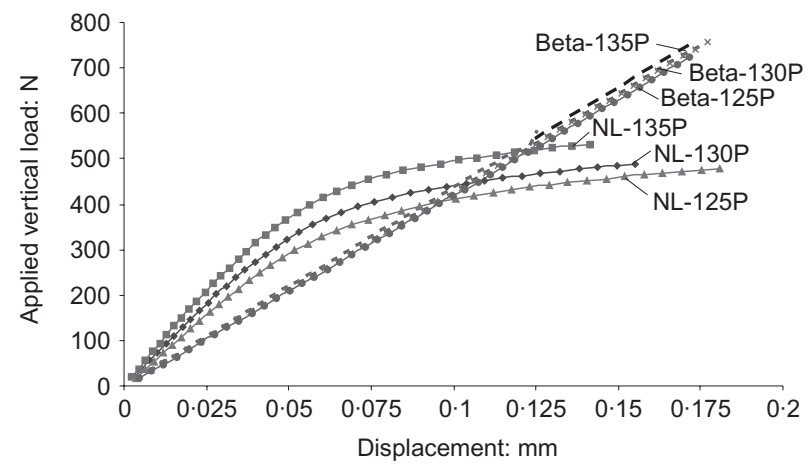

Fig. 15. Load-displacement plot 


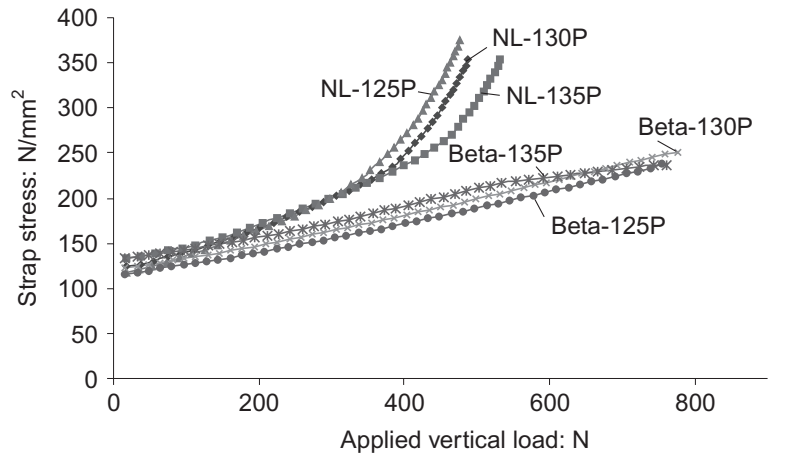

Fig. 16. Strap stress variation with applied load

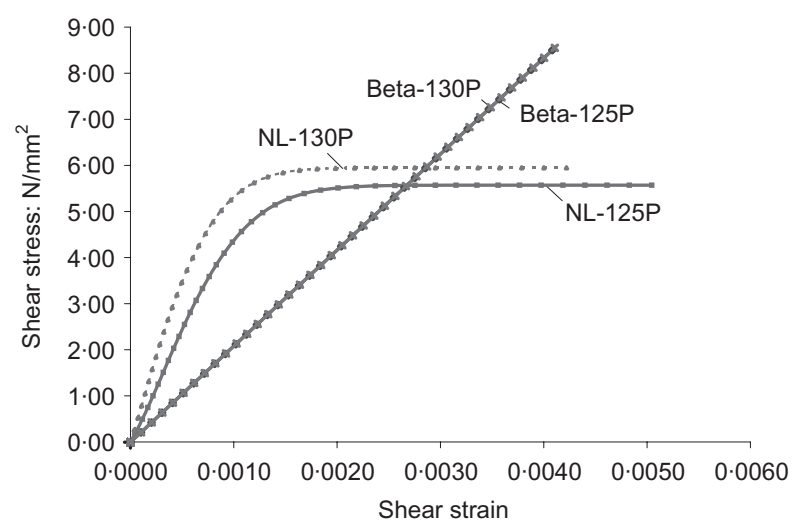

Fig. 17. Shear behaviour in concrete

shows the shear stress variation at an integration point in the concrete, it can be seen that, as the concrete is reaching its peak capacity, all the additional shear forces are transferred to the strap causing a dramatic rise in strap stress. The initial prestress levels influenced the concrete behaviour and hence differences in the concrete behaviour affected the final rise in strap stresses.

For a given ultimate strap stress, these results would imply that the strap in NL-125P would fail before the strap of NL-135P and also the maximum shear load at failure for strap NL-125P will be less than in strap NL135P. In effect the initial prestress level has influenced both the failure of the strap and the peak load sustained by the element. This has been largely a result of the stage at which the concrete reached its peak capacity and all the subsequent loads were transferred to the strap.

In contrast, the stress in the strap in the beta model varies linearly with the strap in Beta-135P reaching a higher stress at a lower load than strap in Beta-125P. The shear stress variation in the concrete shown in Fig. 17 shows the constant nature of the Beta model. In effect the concrete will always carry a certain amount of shear and the rest of the load will be taken up by the strap. Since beta is a constant value this load sharing will always be constant. The small nonlinear variations shown towards the end of the analysis are attributable to the effects of the principal stress angles deviating from the fixed crack angle.

\section{Comments on FE modelling}

The parameters used in the analyses detailed above were for comparative purposes only. In many cases only a single element was used and full-size beam analyses will be required to enable further details of some of the parameters to be quantified.

In developing the nonlinear models for shear, numerous difficulties arose. The main difficulty was the relationship between the shear slip and the normal stress that develops along the crack. Experimental data on the loading range in shear are fairly abundant but very limited results exist for unloading and reloading. Since softening models are used in the FE analysis care had to be taken to ensure that the models did load and unload properly.

The programming technique used in CamFEA was such that a huge volume of data had to be stored for each crack so that a decision on whether the element was loading or unloading or reloading in shear could be taken. These data points served as existing marked points to which subsequent strains were compared and a decision taken as to which direction the analysis should then proceed.

In plasticity formulations, where similar cycles of loading, unloading and reloading occur, yield functions are used and the gradients of these yield functions provide a fairly straightforward criterion for a decision to be taken regarding loading, unloading or reloading. Mathematical formulations such as those used in plasticity are required with regard to fracture in concrete to aid in the implementation of some of the very good advanced models that have been derived. Without more advanced formulations, it is difficult for commercial software to incorporate nonlinear models as the techniques used in CamFEA are prone to errors and spurious unloading and reloading may occur. In contrast, the constant-beta model is simple and very robust. There are no difficulties with the unloading or reloading behaviour and no need to consider the normal stress perpendicular to a crack.

\section{Discussion}

The FE case studies help to explain why the CFRP stresses predicted using conventional FE models were lower than the experimental values in beams strengthened with prestressed CFRP shear straps. In the experimental beams where a strap failed, the measured strap strains increased dramatically in the later stages of testing. This coincided with increased crack opening and a diminishing concrete shear contribution. The FE predictions using a constant-beta model will not, however, capture this effect as the model fails to account for the widening and shearing influence of the cracks on the 
structural stiffness. The constant-beta model is therefore unable to reflect the reduction in the ability of the concrete to carry load as the crack displacements increase and the subsequent increase in the stress in the CFRP reinforcement. An unexpected stress transfer is a particular concern with prestressed straps since the initial prestress reduces the reserve strain capacity that is available after cracking. As a result, it is possible to erroneously miss the potential for failure owing to a strap breaking and indeed, this was the case in the predictions for several of the experimental CFRP strengthened beams. ${ }^{22}$

If ductile reinforcement had been used, the large transfer of stress at the break down of aggregate interlock would lead to a transfer of load to the reinforcement which might result in the yielding of the reinforcement and a certain amount of redistribution. With an FRP reinforcing element, however, there is no yielding and the stress transfer may exceed the ultimate capacity of the reinforcing element leading to instantaneous failure. Hence in the analysis of cracked concrete with highly stressed FRP reinforcement, attempts must be made to predict the actual stresses in both the concrete and the brittle reinforcement.

When investigating the behaviour of existing cracked reinforced concrete structures, the stress transfer during unloading and reloading will also be of importance. The use of crack models which do not take into account the possibility of residual deformations may again underestimate the stress transferred into the reinforcement. Hence, if the shear behaviour is not modelled nonlinearly and no permanent normal or shear crack deformations are allowed then the stiffness predictions would deviate from the real behaviour and the results would be misleading.

The beneficial effect of prestressing and the corresponding enhancement of the inherent concrete shear strength will also not be fully captured when a constant-beta model is used. In particular, the strength gain will not be merely a result of the addition of the strap capacity but there will be an additional benefit owing to aggregate interlock enhancement. This gain in strength seems to be a function of the prestress force in the strap (see Fig. 15).

With the constant-beta method, there is also a difficulty in the selection of an appropriate value for beta. The results have shown such a strong interaction between the stress in the concrete and the crack width that the use of a single beta value would not be able to capture all the possible influences during all phases of loading. Hence, if a single beta value is selected, it is important to bear in mind that stress transfer and stiffness predictions might all be in error. Within DIANA there is a more advanced beta crack model where the value of beta is a function of the crack width. This refinement would potentially give improved results but unfortunately this model was not supported in the version of software used.
The nonlinear models used in CamFEA appeared to be able to predict key features of the stress transfer from the concrete into the strap. However, this program is currently only able to analyse structures with a limited number of elements so it has not yet been possible to model beams using the nonlinear shear cracking models. In addition, it is expected that with a larger number of elements using the advanced nonlinear models, the solutions become more and more complex and indeed convergence may be an issue. One advantage of the constant-beta model is that it is numerically fairly robust.

These initial studies demonstrate the importance of the proper selection of crack models when conducting FE analyses of cracked concrete prestressed with brittle FRP reinforcement.

\section{Conclusions}

The current paper has investigated the selection of normal and shear crack models in a smeared-crack FE analysis of concrete. A benchmarking study of normal crack models showed that the treatment of residual deformations during unloading and reloading was important. Furthermore, the normal crack behaviour must be properly represented in order for the shear behaviour to be correctly modelled since there is a coupling between the normal and shear components in advanced shear crack models. In terms of the shear models, the use of a nonlinear model captures the experimentally observed loss in shear stiffness with increasing loading and also the influence of the normal crack opening and additional prestress force on the behaviour. In contrast, a constant shear retention model only provides a simple approximation of the shear stiffness. While the constant-beta model is numerically robust, it was not able adequately to reflect the behaviour of concrete with a prestressed CFRP reinforcing element. A particular concern was that once extensive cracking has occurred, the stresses in the CFRP reinforcement may well be underestimated when a constant-beta model commonly found in commercial FE programs is used. Indeed, this is consistent with results reported elsewhere where the stresses in external CFRP shear straps were underpredicted during the later stages of loading. ${ }^{2}$

\section{References}

1. Lees J. M., Winistoerfer A. U. and Meier U. External prestressed CFRP straps for the shear enhancement of concrete. ASCE Journal of Composites for Construction, 2002, 6, No. 4, 249-256.

2. Kesse G. Concrete Beams with External Prestressed Carbon FRP Shear Reinforcement. PhD thesis, University of Cambridge, UK, 2003.

3. Bazant Z. P. and Planas J. Fracture and Size Effect in Concrete and Other Quasibrittle Materials. CRC Press, Boca Raton, Florida, 1998. 
4. YankeleVsKy D. Z. and ReinhaRdT H. W. Uniaxial behaviour of concrete in cyclic tension. Journal of Structural Engineering, ASCE, 1989, 115, No. 1, 166-182.

5. Reinhardt H. W., Cornelissen H. A. W. and Hordijk D. A. Tensile tests and failure analysis of concrete. Journal of Structural Engineering, ASCE, 1986, 112, No. 11, 2462-2477.

6. Gopalaratnam V. S. and Shah S. P. Softening response of plain concrete in direct tension. ACI Journal, 1985, 82, No. 3, 310-323.

7. Phillips D. V. and Zhang B. S. Direct tension tests on notched and un-notched plain concrete specimens. Magazine of Concrete Research, 1993, 45, No. 162, 25-35.

8. Yankelevsky D. Z. and Reinhardt H. W. Response of plain concrete to cyclic tension. ACI Materials Journal, 1987, 84, No. 5, 365-373.

9. Paulay T. and Loeber P. J. Shear transfer by aggregate interlock. ACI Special Publication, SP-42, 1974, 1, 1-14.

10. Houde J. and Mirza M. S. A finite element analysis of shear strength of reinforced concrete beams. ACI Special Publication, $S P-42,1974,1,103-128$.

11. Divakar M. P., Fafitis A. and Shah S. P. Constitutive model for shear transfer in cracked concrete. Journal of Structural Engineering, ASCE, 1987, 113, No. 5, 1046-1062.

12. Yoshikawa H., Wu Z. and Tanabe T. Analytical model for shear slip of cracked concrete. Journal of Structural Engineering, ASCE, 1989, 115, No. 4, 771-788.

13. Walraven J. C. Fundamental analysis of aggregate interlock. Journal of the Structural Division, ASCE, 1981, 107, No. ST11, 2245-2271.

14. Reinhardt H. W. and Walraven J. C. Cracks in concrete subject to shear. Journal of the Structural Division, ASCE, 1982, 108, No. ST1, 207-225.
15. TAYLOR H. J. P., Investigation of the forces carried across cracks in reinforced concrete beams in shear by interlock of aggregate. Cement and Concrete Association, Technical Report, (42.447), November 1970

16. Tassios T. P. and Vintzeleou E. N. Concrete-to-concrete friction. Journal of Structural Engineering, ASCE, 1987, 113, No. $4,832-849$.

17. Jimenez R., White R. N. and Gergely P. Cyclic shear and dowel action models in RC. Journal of the Structural Division, ASCE, 1982, 108, No. ST5, 1106-1123.

18. Heuze F. E. and Barbour G. T. New models for rock joints and interfaces. Journal of the Geotechnical Engineering Division, ASCE, 1982, 108, No. GT5 757-776.

19. DE Borst R. Computational aspects of smeared crack analysis Computational Modelling of RC Structures (Hinton E. and Owen D. R. J. (eds)), Chapter 2, Pineridge Press, Swansea, 1986, pp. 44-81.

20. Diana, DIANA User's Manual, Nonlinear Analysis, Release 7, TNO Building and Construction, Netherlands, 2000.

21. GAJER G. and Dux P. Crack band based model for FEM analysis of concrete structures. Journal of Structural Engineering, ASCE, 1990, 116, No. 6, 696-1711.

22. Kesse G. and LeEs J. M. Experimental behaviour of beams strengthened in shear with prestressed CFRP Straps. ASCE Journal of Composites for Construction, in press.

Discussion contributions on this paper should reach the editor by 1 November 2007 